\title{
RELAÇÃO ENTRE COMPULSÃO ALIMENTAR E DIETAS RESTRITIVAS COM ESTADO NUTRICIONAL DE ESTUDANTES DOS CURSOS DE NUTRIÇÃO E EDUCAÇÃO FÍSICA
}

\author{
RELATIONSHIP BETWEEN FOOD COMPULSION AND RESTRICTIVE \\ DIETS WITH NUTRITIONAL STATUS OF STUDENTS IN NUTRITION \\ AND PHYSICAL EDUCATION COURSES
}

Danielle Martins Gonçalves $^{1}$
Dórits Gonçalves Andrade ${ }^{2}$
Larissa de Brito Medeiros $^{3}$

RESUMO: Objetivo: Correlacionar a adesão a dietas restritivas e desenvolvimento de compulsão alimentar com estado nutricional de estudantes dos cursos de Nutrição e Educação Física do município de Cajazeiras-PB. Metodologia: A população do estudo foi composta por 45 estudantes com idade entre 18 anos e 40 anos, de ambos os sexos, dos cursos de Nutrição e Educação Física, do município de Cajazeiras-PB, da Faculdade da Santa Maria (FSM) e Faculdade de Filosofia Ciências e Letras (FAFIC). Quanto à coleta de dados, foi realizada uma análise antropométrica para avaliação do estado nutricional e os estudantes foram submetidos a um Questionário de Adesão a Dietas Restritivas e Percepção de Imagem Corporal de autopreenchimento, a fim de avaliar os motivos, tipo e duração de adesão a dietas restritivas e aspectos quanto à auto percepção de imagem corporal. Posteriormente, foram submetidos ao preenchimento da Escala de Compulsão Alimentar Periódica (ECAP) para serem avaliados sintomas ou características que possam estar relacionado ao desenvolvimento da compulsão alimentar. Os dados foram tabulados no programa Microsoft Office Excel® 2016 para a realização da estatística descritiva e apresentados em percentual, média e desvio-padrão. As análises dos questionários foram realizadas no SPSS (versão 24). Foi utilizado o teste inferencial correlação de Pearson entre os dados obtidos dos questionários e o IMC. O critério de significância estatística adotado foi de $p \leq 0,05$. Resultados: A partir dos resultados obtidos, foi possível perceber que grande parte dos estudantes apresentava insatisfação corporal, com correlação direta com maior

\footnotetext{
${ }^{1}$ Pós-Graduanda em Nutrição Clínica Funcional da Faculdade Santa Maria.

${ }^{2}$ Pós-Graduanda em Nutrição Clínica Funcional da Faculdade Santa Maria.

${ }^{3}$ Docente do Curso de Nutrição da Faculdade Santa Maria.
} 
IMC e maior pontuação da ECAP. Além disso, a grande adesão a dietas restritivas teve relação diretamente proporcional com o risco de desenvolvimento de compulsão alimentar. Esses resultados corroboram com estudos anteriores que apontam a relação entre dietas restritivas e compulsão alimentar, bem como, a maior suscetibilidade dos estudantes da área da saúde para ao desenvolvimento de distúrbios alimentares, principalmente dos cursos de Nutrição e Educação Física. Conclusão: Os estudantes dos cursos de Nutrição e Educação Física tem risco de desenvolver transtornos alimentares e de percepção da imagem corporal, aderindo a dietas restritivas sem acompanhamento profissional, com o intuito de adequação em padrões estéticos muitas vezes associados às suas capacidades intelectual e profissional. Assim, sugere-se uma investigação de outras variáveis que possam estar envolvidas nesse comportamento, com o objetivo de identificar riscos e potencializar intervenções preventivas no âmbito acadêmico.

Palavras chave: Dieta Redutora. Transtorno da Compulsão Alimentar. Saúde do Estudante. Nutrição. Educação Física e treinamento.

ABSTRACT: Objective: Correlate adherence to restrictive diets and development of binge eating with nutritional status of students of Nutrition and Physical Education courses in the municipality of Cajazeiras-PB. Methodology: The study population consisted of 45 students aged between 18 and 40 years old, of both sexes, from the Nutrition and Physical Education courses, in the municipality of Cajazeiras-PB, Faculdade da Santa Maria (FSM) and Faculdade of Philosophy Sciences and Letters (FAFIC). As for data collection, an anthropometric analysis was carried out to assess nutritional status and students were submitted to a Self-Filling Compliance Questionnaire and Perception of Body Image, in order to assess the reasons, type and duration of adherence to restrictive diets and aspects of self-perception of body image. Subsequently, they were submitted to the Periodic Eating Compulsion Scale (ECAP) to assess symptoms or characteristics that may be related to the development of binge eating. The data were tabulated in the Microsoft Office Exce/ $\mathbb{R}$ 2016 program to perform descriptive statistics and presented in percentages, mean and standard deviation. The analysis of the questionnaires was carried out in SPSS (version 24). The inferential Pearson correlation test was used between the data obtained from the questionnaires and the BMI. The criterion for statistical significance adopted was $p \leq 0.05$. Results: From the results obtained, it was possible to notice that most of the students had body dissatisfaction, with a direct correlation with higher BMI and higher ECAP score. In addition, the high adherence to restrictive diets was directly proportional to the risk of developing binge eating. These results corroborate with previous studies that point out the relationship between restrictive diets and binge eating, as well as, the greater susceptibility of students in the health area to the development of eating disorders, especially in Nutrition and Physical Education courses. Conclusion: Students of the Nutrition and Physical Education courses are at risk of developing eating disorders and perception of body image, adhering to restrictive diets without professional monitoring, in order to adapt to aesthetic standards often associated with their intellectual and professional capacities. Thus, an investigation of other variables that may be involved in this 
behavior is suggested, in order to identify risks and enhance preventive interventions in the academic field.

Keywords: Diet, Reducing. Binge-Eating Disorder. Student Health. Nutrition. Physical Education and Training. 


\section{INTRODUÇÃO}

Hábitos alimentares saudáveis estão relacionados com a quantidade e com a qualidade nutricional dos alimentos ingeridos. A diminuição do consumo de frutas, verduras, legumes e hortaliças têm sido cada vez mais observadas na dieta da população, associado ao aumento da ingestão de alimentos gordurosos industrializados e ricos em açúcar. Essa transição alimentar tem causado um impacto negativo na saúde da população, pois tem sido consequência das mudanças comportamentais para um estilo de vida moderno, no qual os indivíduos buscam praticidade, optando por alimentos calóricos e de baixa qualidade nutricional (COSTA et al., 2018).

Essas mudanças alimentares resultaram no aumento de morbidade $\mathrm{e}$ mortalidade associadas ao estilo de vida moderno (RECINE; CARVALHO, 2015), como a obesidade, cada vez mais prevalente na população (MORENO et al., 2014). Os principais gatilhos para o desenvolvimento da obesidade são alimentação desequilibrada e o sedentarismo, decorrentes da rotina contemporânea (FERNANDES et al., 2020). Em contrapartida, nos últimos anos, o corpo magro e com baixíssimo percentual de gordura, modelo estético antagônico da maioria da população, tem sido imposto pela mídia e sociedade, gerando descontentamento da auto percepção corporal de muitas pessoas (PENZ; BOSCO; VIEIRA, 2008).

Diante da atual conjuntura, a procura por métodos que acarretem em emagrecimento rápido e adequação aos padrões corporais impostos pela mídia e sociedade têm se tornado cada vez mais frequente, resultando em sérios riscos à saúde, por utilização de métodos de perda de peso insustentáveis sem o acompanhamento adequado por profissionais qualificados (ABREU et al., 2013). Atualmente, muitas pessoas são influenciadas a aderir a dietas altamente restritivas, divulgadas por figuras públicas que garantem o alcance de corpos atraentes de maneira rápida, sem qualquer respaldo científico, comprometendo a saúde por não 
atingirem as recomendações nutricionais adequadas (PERINAZZO; ALMEIDA, 2010).

O acesso a essas dietas tem se tornado cada vez mais corriqueiro, com o mesmo propósito de perda de peso imediata, através do incentivo de redução drástica ou exclusão de nutrientes essenciais para o desenvolvimento e a manutenção do organismo. Dietas com baixo teor de carboidratos e alto consumo de proteínas, jejuns prolongados e dietas hiperlipídicas sem acompanhamento de profissionais são alguns exemplos de imprudências cometidas por indivíduos que buscam o emagrecimento de forma brusca (MELANSON et al., 2012).

Alguns estudos mostram que restrições alimentares estão relacionadas com posterior desenvolvimento de compulsão alimentar (CA), caracterizada pelo consumo excessivo de alimento em curto período de tempo associado à sensação de perda de controle (DUARTE et al., 2015). Tal comportamento prejudica a ingestão e absorção dos alimentos afetando a saúde física e o desempenho psicossocial (ALVARENGA; SCAGLIUSI; PHILIPPI, 2011).

Estudantes dos cursos Nutrição e Educação Física possuem uma maior predisposição ao desenvolvimento de transtornos alimentares quando comparados a estudantes de cursos de outras áreas (MAHN; LORDLY, 2015). Entretanto, os estudos não conseguiram elucidar se essa maior predisposição está relacionada com o maior conhecimento desses estudantes sobre alimentação e influência de suas experiências no controle de peso, ou se está relacionada à crença de que a boa aparência é fundamental para o futuro sucesso profissional (FREITAS et al., 2017).

Diante desse contexto, o presente trabalho teve como objetivo correlacionar a adesão a dietas restritivas e o risco de desenvolvimento de compulsão alimentar com o estado nutricional em estudantes dos cursos de Nutrição e Educação Física no município de Cajazeiras-PB. Essa pesquisa possibilita identificar os riscos psicológicos e nutricionais aos quais esses estudantes estão vulneráveis, evidenciando e fornecendo novos dados para intervenção de outros profissionais. 


\section{METODOLOGIA}

Trata-se de um estudo epidemiológico com delineamento transversal e abordagem quantitativa, apresentando caráter exploratório e descritivo.

A população do estudo foi composta de estudantes de todos os períodos dos cursos de Nutrição e Educação Física, do município de Cajazeiras-Paraíba, da Faculdade da Santa Maria (FSM) e Faculdade Filosofia Ciências e Letras (FAFIC). A amostragem foi não probabilística por conveniência composta por 45 alunos das instituições FSM e FAFIC com idade entre 18 anos e 40 anos de ambos os sexos, sendo 25 do curso de Nutrição e 20 do curso do curso de Educação Física.

Foram excluídos da pesquisa indivíduos que faziam uso de medicamentos ansiolíticos ou que tinham diagnóstico de distúrbios psiquiátricos.

O presente estudo foi aprovado pelo Comitê de Ética em da Faculdade Santa Maria ( $n^{\circ}$ do parecer 2.556.309) (ANEXO) e conduzido com base nos requisitos e critérios prescritos pela Resolução $n^{\circ}$ 466/2012, do Conselho Nacional de Saúde (CNS), a qual versa sobre a ética em pesquisa com seres humanos.

Os estudantes foram submetidos a um Questionário de Adesão a Dietas Restritivas e Percepção de Imagem Corporal de autopreenchimento, por meio de questões objetivas de autoafirmação. A partir do questionário, foram analisados os motivos, tipo e duração de adesão a dietas restritivas, além disso, foram avaliados aspectos quanto à auto percepção de imagem corporal. O questionário passou por validação do Colegiado do curso de Nutrição da Faculdade Santa Maria.

Simultaneamente à aplicação do questionário citado acima, para avaliação de compulsão alimentar, foi utilizada e aplicada a Escala de Compulsão Alimentar Periódica (ECAP) traduzida e adaptada da Binge-Eating Scale (GORMALLY et al, 1982) por Freitas e colaboradores (2001), com intuito de avaliar o grau de comportamentos compulsivos dos estudantes em relação à alimentação.

Em seguida à aplicação dos questionários, os participantes da pesquisa foram submetidos à avaliação nutricional. Com uma balança antropométrica digital (Tech Line TEC-130) e fita inelástica (Sanny), foram aferidos peso, estatura, 
circunferências da cintura (CC) e do quadril (CQ) (CAVALCANTI et al., 2009; SAMPAIO, 2012). Para identificação do estado nutricional foram utilizados dois Índices: Índice de Massa Corporal (IMC), obtido a partir do valor do peso dividido pela altura $^{2}$ (THOMAZ; SILVA; COSTA, 2013); e razão cintura quadril (RCQ), considerada alterada quando seu resultado se encontra acima de 1 no público masculino e 0,85 no público feminino (VELOSO; SILVIA, 2010).

Os dados foram tabulados no programa Microsoft Office Excelß 2016 para a realização da estatística descritiva e apresentados em percentual, média e desviopadrão. As análises dos questionários foram realizadas no SPSS (versão 24). Foi utilizado o teste inferencial correlação de Pearson entre os dados obtidos dos questionários e o IMC. O critério de significância estatística adotado foi de $p \leq 0,05$.

\section{RESULTADOS E DISCUSSÃO}

Para realização da pesquisa foram avaliados 45 estudantes com idade média de $22,13 \pm 5,61$ anos, sendo $51,1 \%$ do sexo masculino e $48,9 \%$ do sexo feminino, dos quais 55,6\% cursam o curso de Nutrição do terceiro ao sétimo período, e 44,4\% cursam Educação Física do primeiro ao quinto período.

A partir da análise do perfil dos participantes, é possível perceber na Tabela 1 que a média do IMC dos participantes do estudo encontra-se no estado nutricional de eutrofia, de acordo com Fagundes et al. (2004), bem como, a média da CC encontra-se abaixo de $80 \mathrm{~cm}$ e a razão cintura quadril abaixo de 0,8 , não demonstrando risco de desenvolvimento de doenças cardiovasculares e morbidades (VELOSO; SILVIA, 2010).

Tabela 1 - Média das variáveis do estado nutricional.

\begin{tabular}{|c|c|c|c|c|}
\hline & PESO (Kg) & IMC (Kg/m $\left.{ }^{2}\right)$ & $C C(\mathrm{~cm})$ & RCQ \\
\hline Média & 65,08 & 23,46 & 75,22 & 0,75 \\
\hline Desvio padrão & 11,96 & 2,93 & 9,55 & 0,07 \\
\hline
\end{tabular}


Apesar do perfil do estado nutricional dos participantes do estudo apresentarse adequado, há uma grande insatisfação corporal pelos estudantes dos cursos de Nutrição e Educação Física entrevistados. A partir da Tabela 2, pode-se constatar esse fato, pois é observada uma correlação inversamente proporcional entre a satisfação corporal e o IMC, mesmo os indivíduos estando eutróficos. Além disso, o desejo de ser magro (a) está diretamente correlacionado com o IMC, demonstrando que quanto maior o IMC do estudante, maior a vontade de estar com o corpo magro (Tabela 2).

Tabela 2 - Correlação entre IMC e Pontuação do ECAP com percepção de imagem corporal e adesão a dietas restritivas.

\begin{tabular}{|c|c|c|}
\hline $\begin{array}{l}\text { QUESTIONÁRIO DE ADESÃO A DIETAS RESTRITIVAS E } \\
\text { PERCEPÇÃO DE IMAGEM CORPORAL }\end{array}$ & IMC & $\begin{array}{l}\text { PONTUAÇÃO } \\
\text { ECAP }\end{array}$ \\
\hline Você é satisfeito (a) com o seu corpo & 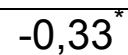 & $-0,49^{* *}$ \\
\hline Você gostaria de mudar algo em seu corpo & 0,05 & 0,21 \\
\hline Você gostaria de ser mais magro & $0,51^{* *}$ & $0,25^{*}$ \\
\hline Você já seguiu alguma dieta por conta própria & $0,28^{*}$ & $0,26^{*}$ \\
\hline $\begin{array}{l}\text { Você já seguiu dietas com acompanhamento de um } \\
\text { profissional }\end{array}$ & 0,18 & 0,19 \\
\hline Você obteve resultado satisfatório com a dieta & 0,09 & $-0,16$ \\
\hline Você já seguiu mais de um tipo de dieta & $0,25^{*}$ & 0,12 \\
\hline $\begin{array}{l}\text { Você já excluiu algum tipo de alimento da sua alimentação } \\
\text { por medo de engordar }\end{array}$ & 0,14 & 0,04 \\
\hline Você já se sentiu mal por não ter o corpo que queria & $-0,03$ & $0,33^{*}$ \\
\hline $\begin{array}{l}\text { Você se submeteria a métodos radicais para ter o corpo que } \\
\text { deseja }\end{array}$ & 0,11 & 0,23 \\
\hline $\begin{array}{l}\text { Você já se sentiu pressionada (o) para estar em boa forma } \\
\text { por causa do seu curso }\end{array}$ & 0,05 & $0,37^{* *}$ \\
\hline $\begin{array}{l}\text { Depois que você entrou na faculdade teve desejo de mudar } \\
\text { de corpo }\end{array}$ & 0,04 & 0,14 \\
\hline $\begin{array}{l}\text { Você já fez dietas restritas para conseguir o corpo que } \\
\text { deseja }\end{array}$ & $0,29^{*}$ & $0,25^{*}$ \\
\hline $\begin{array}{l}\text { Se arrepende de ter aderido a dietas restritas para ter o } \\
\text { corpo que deseja }\end{array}$ & 0,01 & 0,12 \\
\hline $\begin{array}{l}\text { Você se incomoda quando dizem que você está acima do } \\
\text { peso }\end{array}$ & $0,34^{*}$ & 0,21 \\
\hline
\end{tabular}
ECAP - Escala de Compulsão Alimentar Periódica.

Notas: Correlação bisserial por ponto. ${ }^{*}$ A correlação é significativa no nível 0,05 (unicaudal); ${ }^{* *}$. A correlação é significativa no nível 0,01 (unicaudal). 
Respostas sim Correlação de Spearman. Correlação diretamente proporcional $=$ sinal positivo; Correlação inversamente proporcional $=$ sinal negativo . ** $p<0,001$.

Esses achados são bastante preocupantes, pois foi visto também no presente trabalho que, quanto maior o IMC, mais o indivíduo se incomoda se outras pessoas comentam em relação ao seu peso (Tabela 2). Isso pode estar bem associado ao estereótipo das futuras profissões, pois tem se tornado crescente o número de estudantes desses cursos insatisfeitos com seu próprio corpo, aumentando a busca por magreza pela maioria do público feminino e o corpo musculoso pelo masculino (MIRANDA et al., 2012). Essas atitudes colaboram para o desenvolvimento de comportamentos inapropriados em relação à alimentação e ao controle sobre o próprio peso, propiciando a adesão de dietas altamente restritivas, excluindo refeições ou alimentos fundamentais para o funcionamento do organismo, afim de alcançar o corpo assumido como "perfeito" (AINETT et al., 2017).

A maioria dos estudantes $(57,8 \%)$ já aderiu a dietas por decisão própria sem acompanhamento profissional. Essa adesão a dietas, muitas vezes restritivas, apresentou correlação diretamente proporcional com o IMC e pontuação da Escala de Compulsão Alimentar (ECAP) aplicada neste estudo (Tabela 2). Muitas vezes, a adesão a essas dietas é motivada para alcançar um padrão corporal imposto pela sociedade relativo à sua futura profissão. Esses estudantes, por escolherem profissões que exercem uma função essencial na conscientização de hábitos saudáveis que podem influenciar na transformação da imagem corporal, são cobrados desde a sua formação acadêmica a estarem dentro dos padrões estéticos atuais. A sua aparência torna-se um indicador de elevada capacidade e intelecto, pressionando-os a apresentarem uma forma física atraente como comprovação da sua eficiência laboral (BANDEIRA et al., 2016).

A partir da Tabela 2, pode-se observar correlação entre a insatisfação corporal e adesão a dietas, como já discutido. Entretanto, é possível constatar também, correlação diretamente proporcional entre a insatisfação corporal e a adesão a dietas com uma maior pontuação da ECAP, indicando que esses estudantes são indivíduos que apresentam risco de desenvolverem transtornos alimentares, como a compulsão. 
Segundo Oliveira et al. (2019), a adesão corriqueira a dietas bastante restritivas, principalmente realizadas por conta própria, está relacionada ao maior risco de desencadeamento de transtornos alimentares em jovens universitários. Contudo, por não conseguirem manter essas dietas em longo prazo, muitas vezes, ocorre o desenvolvimento de compulsão alimentar (CA) (VEENSTRA; DE JONG, 2010), caraterizada como alta ingestão de alimento em um curto espaço de tempo, associada à sensação de angústia e descontrole sobre o que e o quanto come (DSMV, 2014).

Assim como a presente pesquisa demonstrou, indivíduos que relatam altos e intermediários níveis de insatisfação corporal são propícios a desenvolver CA, e a dieta restritiva tem sido comprovada como forte propulsora no desencadeamento deste transtorno, se tornando alvo de diversos estudos (JOHNSON; PRATT; WARDLE, 2012; KUIJER et al., 2008; SOUTO; FERRO-BUCHER, 2006). Já é bem estabelecido na literatura que a recorrência da restrição dietética relacionada ao desenvolvimento de CA ocorre tanto em obesos, como em indivíduos com peso normal (COFFINO; ORLOFF; HORMES, 2016).

Em concordância com o presente estudo, algumas pesquisas têm comprovado que o risco de incidência de transtornos alimentares tem se tornado cada vez mais comum entre os estudantes dos cursos de Nutrição e Educação Física, além da preocupação excessiva com o controle de peso com a finalidade de estar entre os padrões estéticos impostos pela mídia, fato bastante alarmante e que traz uma grande reflexão quanto ao tema, demonstrando a grande relevância e contribuição do presente trabalho (REIS et al., 2014; LAUS; MOREIRA; COSTA, 2009).

\section{CONCLUSÃO}

A partir do presente estudo é possível observar que, apesar do perfil do estado nutricional dos participantes do estudo apresentar-se adequado, houve um número relevante de estudantes dos cursos de Nutrição e Educação Física com 
insatisfação corporal, apresentando uma correlação significativa diretamente proporcional com a adesão a dietas restritivas sem acompanhamento profissional adequado. Além disso, essa insatisfação corporal, principalmente associada com as futuras profissões, está diretamente relacionada com comportamentos de compulsão alimentar.

Portanto, pode-se perceber que acadêmicos da área de nutrição e educação física são indivíduos vulneráveis à adesão de dietas restritivas com o intuito de adequação em padrões estéticos muitas vezes associados às suas capacidades intelectual e profissional. Essa adesão está associada diretamente com o risco de desenvolvimento de compulsão alimentar, fato já evidenciado em outros estudos. Assim, a atual pesquisa traz resultados importantes quanto aos riscos que esse público tem em desenvolver transtornos alimentares e de percepção da imagem corporal, sugerindo investigação de outras variáveis que possam estar envolvidas, com o objetivo de identificar riscos e potencializar intervenções preventivas no âmbito acadêmico. 


\section{REFERÊNCIAS BIBLIOGRÁFICAS}

ABREU, E. S. de et al. Parâmetros nutricionais de dietas anunciadas na imprensa leiga destinada ao público masculino e feminino. Revista Ciência \& Saúde, v. 31, n. 1, p. 96-103, 2013.

AINETT, W. S. de O. et al. FATORES ASSOCIADOS À INSATISFAÇÃO COM A IMAGEM CORPORAL EM ESTUDANTES DE NUTRIÇÃO. Revista Brasileira de Obesidade, Nutrição e Emagrecimento, São Paulo., v. 11, ed. 62, p. 75-85, 2017.

ALVARENGA, M. S; SCAGLIUSI, F. B; PHILIPPI, S. T. Comportamento de risco para transtorno alimentar em universitárias brasileiras. Revista de Psiquiatria Clínica, v. 38, n. 1, p.3-7, 2011.

BANDEIRA, Y. E. R. et al. Avaliação da imagem corporal de estudantes do curso de Nutrição de um centro universitário particular de Fortaleza: Avaliação da imagem corporal. Jornal Brasileiro Psiquiatria. Fortaleza, p. 168-173. 28 mar. 2016.

CAVALCANTI, C. L. et al. Prevalência de doenças crônicas e estado nutricional em um grupo de idosos brasileiros. Revista Saúde Pública, v. 11, n. 6, p. 865-877, 2009.

COFFINO, J. A.; ORLOFF, N. C.; HORMES, J. M. Dietary Restraint Partially Mediates the Relationship between Impulsivity and Binge Eating Only in Lean Individuals: The Importance of Accounting for Body Mass in Studies of Restraint. Frontiers in Psychology, v. 7, 2016.

COSTA, C. S. et al. Comportamento sedentário e consumo de alimentos ultraprocessados entre adolescentes brasileiros: Pesquisa Nacional de Saúde do Escolar (PENSE), 2015. Caderno Saúde Pública, Rua Marechal Deodoro 1160, 3o andar, Pelotas, v. 34, ed. 3, p. 1-10, 2018.

DSMV. MANUAL DIAGNÓSTICO E ESTATÍSTICO DE TRANSTORNOS MENTAIS [RECURSO ELETRÔNICO]: DSM-5 / [American Psychiatric Association; tradução: Maria Inês Corrêa Nascimento... et al.]; revisão técnica: Aristides VolpatoCordioli ... [et al.]. - 5. ed. - Dados eletrônicos. - Porto Alegre: Artmed, 2014.

DUARTE et al. Expanding binge-eating assessment: Validity and screening value of the binge eating scale in women from the general population. Eating Behaviors, v. 18, p. 41-47, 2015.

FAGUNDES, A. A. et al. Vigilância Alimentar e Nutricional - SISVAN: Orientações básicas para a coleta, o processamento, a análise de dados e a informação em serviços de saúde. Brasília - DF: Ministério da Saúde, 2004. 122 p.

FERNANDES, C. A. M. et al. Prevalência de sedentarismo em adultos obesos e sobrepesados. Revista Enfermagem Atual, v. 93, ed. 30, p. 243-247, 2020.

FREITAS, D. et al. Eating behaviour among nutrition students and social desirability as a confounder. Appetite, v. 113, p. 187-192, 2017.

GORMALLY, J. et al. The assessment of binge eating severity among obese persons. Addictive Behaviors, v. 7, n. 1, p. 47-55, 1982.

JOHNSON, F.; PRATT, M.; WARDLE, J. Dietary restraint and self-regulation in eating behavior. International Journal of Obesity, v. 36, n. 5, p. 665- 674, 2012.

KUIJER, R. et al. Dieting as a case of behavioural decision making: does self-control matter? Appetite, v. 51, n. 3, p. 506- 511, 2008. 
LAUS, M. F; MOREIRA, R. C. M; COSTA, T. M. B. Diferenças na percepção da imagem corporal, no comportamento alimentar e no estado nutricional de universitárias das áreas de saúde e humanas. Revista Psiquiatria, v. 31, n. 3, p. 192-196, 2009.

MAHN, H. M., LORDLY, D. A review of eating disorders and disordered eating amongst nutrition students and dietetic professionals. Canadian Journal of Dietetic Practice and Research, v. 76, n. 1, p. 38-43, 2015.

MELANSON, K. J. et al. Body composition, dietary composition, and components of metabolic syndrome in overweight and obese adults after a 12-week trial on dietary treatments focused on portion control, energy density, or glycemic index. Nutrition Journal, p. 1-9, 2012.

MIRANDA, V. P. N. et al. Insatisfação corporal em universitários de diferentes áreas de conhecimento. Jornal Brasileiro Psiquiatria. Juiz de Fora, Mg, Brasil, p. 25-32. 25 jan. 2012.

MORENO, B. et al. Comparison of a very low-calorie-ketogenic diet with a standard low-calorie diet in the treatment of obesity. Springer Science business Media, v. 47, n. 3, p. 1-13, 2014.

OLIVEIRA, J. et al. Prevalência de comportamentos de risco para transtornos alimentares e uso de dieta "low-carb" em estudantes universitários. Jornal Brasileiro Psiquiatria, São Paulo, SP, Brasil., v. 64, ed. 4, p. 183-190, 2019.

PENZ, L. R; BOSCO, S. M; VIEIRA, J. M. Risco para desenvolvimento de transtornos alimentares em estudantes de Nutrição. Scientia Medica, v. 18, p. 124-128, 2008.

PERINAZZO, C; ALMEIDA, J. C. Composição nutricional de dietas para emagrecimento divulgadas em revistas não científicas. Revista do hospital de clinicas e da Faculdade de medicina publica, v. 30, n. 3, 2010.

RECINE, E; LEÃO, M; CARVALHO, M. F. O papel do nutricionista na atenção primária á saúde. 3. Ed. Brasilia - DF: Conselho Federal de Nutricionista, 3.ed, 2015.

REIS, J. A. et al. Fatores associados ao risco de transtornos alimentares entre acadêmicos da área de saúde. Revista Gaúcha de Enfermagem, v. 35, ed. 2, p. 73-78, 2014.

SAMPAIO, L. R. Avaliação Nutricional. Salvador: Editora da Universidade Federal da Bahia, 162 p. 9. 2012.

SOUTO, S.; FERRO-BUCHER, J. S. N. Práticas indiscriminadas de dietas de emagrecimento e o desenvolvimento de transtornos alimentares. Revista de Nutrição de Campinas, v. 19, n. 6 , p. 693-704, 2006.

THOMAZ, P. M. D; SILVA, E. F.; COSTA, T. H. M. Validade de peso, altura e índice de massa corporal autorreferidos na população adulta de Brasília. Revista Brasil Epidemiologia, Brasília- DF, v. 16, n. 1, p. 157-169, 2013.

VELOSO, H. J. F; SILVA, A. A. M. Prevalência e fatores associados à obesidade abdominal e ao excesso de peso em adultos maranhenses. Revista Brasil Epidemiologia, v.13, n.3, p.400412, 2010.

VEENSTRA, E. M.; DE JONG, P. J. Restrained eaters show enhanced automatic approach tendencies towards food. Appetite, v. 55, n.1, p. 30-36, 2010. 


\section{ANEXO - PARECER DO COMITÊ DE ÉTICA EM PESQUISA}

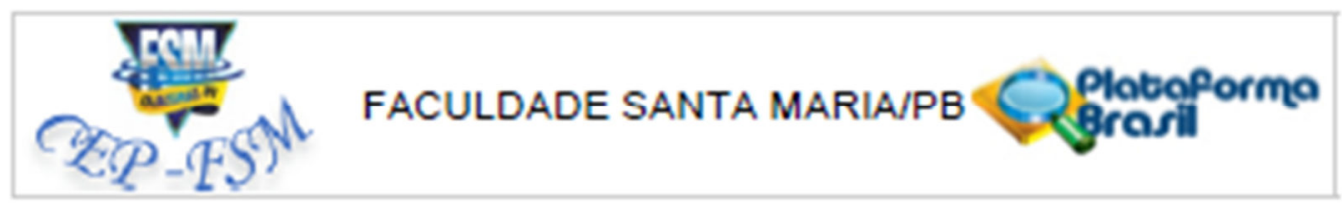

\section{PARECER CONSUBSTANCIADO DO CEP}

\section{DADOS DO PROJETO DE PESQUISA}

TItulo da Pøsqulaa: ASSOCIAÇÅO ENTRE COMPULSÄO ALIMENTAR E DIETAS RESTRITIVAS EM ESTUDANTES DOS CURSOS DE NUTRIÇAO E EDUCAÇÅO FISICA

Perqulaador: LARISSA DE BRITO MEDEIROS

Area Tematuca:

Versao: 1

CAAE: 84970918.1.0000.5180

Inatitulçao Proponente: Facuidade Santa Marial FSM /P8

Patrocinador Prindpal: Financiamento Proprio

\section{DADOS DO PARECER}

Numero do Parecer: 2.556.309

Apresentacaso do Projeto:

Introduç5: Atuaimente a procura por metodos para em emagrecimento rapido e adequaça a0s padrbes corporais impostos pela midla e sodedade

tem se tomado cada vez mals frequente. Multas pessoas săo influencladas a aderir a dletas altamente restrivas. porem alguns estudos mostram

que restriçbes allmentares est5̆o relacionadas com malor probabilldade do desenvoivimento de posterior compuls50 allmentar, caracterizada pelo

consumo excessivo de almento em curto periodo de tempo associado com a sensaçato de perda de controle. Estudantes dos cursos Nutriçato e

Educaç50 Fisica possuem uma malor predsposiç50 a0 desenvolvimento de transtornos allmentares que pode estar relacionado a crença de que a

boa aparenda e indamental para o futuro sucesso pronssional. Oojetivo: Correlacionar a adesło a dletas restritivas com o desenvolvimento de

compulsto allmentar e seus impactos no estado nutricional de estudantes dos cursos de Nutriçăo e Educaçăo Fislca do municiplo de Cajazelras-PB.

Metodologla: Trata-se de um estudo epidemiologlo com delineamento transversa, abordagem quantitativa. com carater expioratorio e descrituo. A

populaçato do estudo sera composta de estudantes com idade entre 18 anos e 40 anos, de ambos 05 sexos, de todos 0 periodos dos cursos de

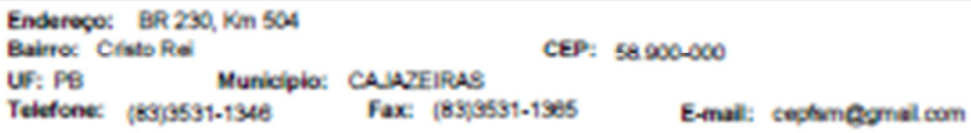




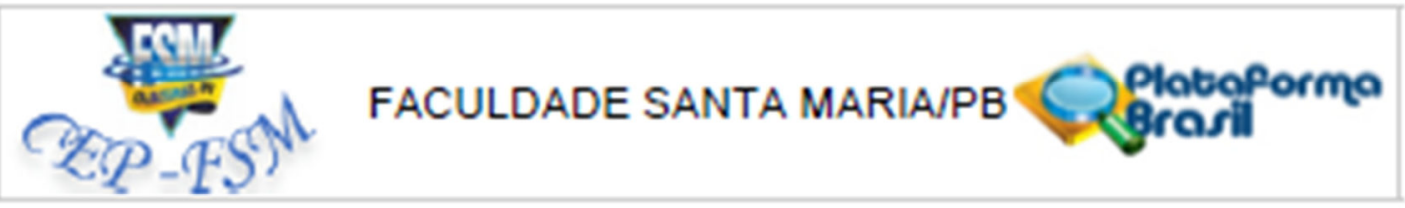

Certhask do Pavear 2.500 .300

Nutrlçăo e Educaçăo Fislca, do munlclplo de Cajazelras-PB, da Faculdade da Santa Marla (FSM) e Faculdade de Fllosonia Clenclas e Letras

(FAFIC). Serdo coletados, mediante a assinatura do Termo de Consentmento LIvre e Esclarecido, dados para a analise do estado nutriciona. $O 6$

estudantes tambem serăo submetdos a um Questionano de Adesto a Dletas Restrituas e Percepç5̆o de Imagem corporal de autopreenchimento, a

im de avallar os motivos, tipo e duraçă de adesto a detas restrituas e aspectos quanto a auto percepçă de Imagem corporal. Posteriormente,

serăo submetdos a preenchimento da Escala de Compulsł̆o Allmentar Pertodlca para ser avallados sintomas ou caracteristicas que possam estar

relacionado ao desenvolvimento da compusto almentar. Ap0s a coleta, o banco de dados e as analses serdo realzados no SPSS (versto 24).

Alem de estatistcas descrituas de frequencia e meddas de tendencla central e de dspersao, sera utilzado o teste de qui-quadrado de Pearson $(x)$ )

e analse de vaniancia ANOVA ou Kruskal-walls, dependendo da distribulçăo dos dados, que sera analisada por melo do teste de Kolmorgororsmimov.

O criterio de signincanda estatistica adotado sera de p0,05. Resultados esperados: Essa pesquisa possibillitara Identifcar 05 riscos

psicologicos e nutitionais aos quals os estudantes dos cursos de Nutriç5o e Educaç5o Fisica da didade de Cajazelras +88 estäo vuineravels,

evidenclando e fornecendo novos dados de como as futuras profssסles podem atetar a saude fisica e mental desse pubilico.

Objetivo da Pesqulsa:

Oojetvo Primano:

Correladionar a ades\$o a dletas restritvas com o desenvowimento de compusto allmentar e seus impactos no estado nutriciona de estudantes dos

cursos de Nutriçăo e Educaçäo Fisica do muriciplo de Cajazeiras-PB,

Cojetvo secundario:

- Avallar o estado nutricional; Avallar a adeşo a dletas restrtilvas; - Avallar a presença de compulş5o almentar: Relacionar o impacto de

dletas restritivas no estado nutricional e desenvolvimento de compuisalo allmentar nos estudantes selecionados.

Endereco: BR $230, \mathrm{Km} 504$

Bairrec Cisto Re

U: PB Municipio: CALZEIRAS

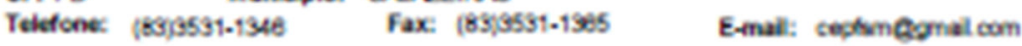




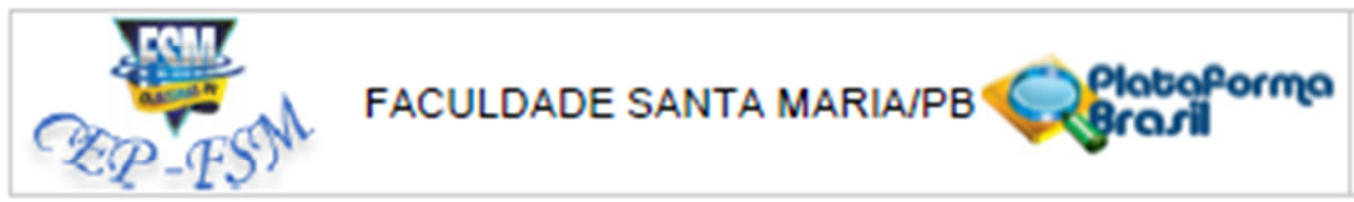

Centhasks do Pavear 2.006 .306

\section{Avallaçso dos Flscos $\theta$ Beneficlos:}

Riscos:

S50 previstos riscos minimos previsivels para quem se submeter a coleta dos dados, tendo em vista tratarse apenas de respostas a questionario e

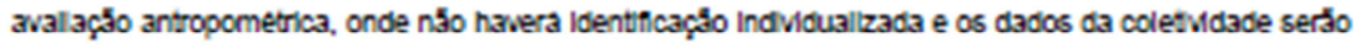
tratados com padrbes evcos (contome

Resoluçăo CNS 466/12) e clentincos. Entretanto, nesta pesquisa, ha possiblidade do participante ter descontortos causados pela aplicaçăo dos

Instrumentos de coletas de dados e possivels constrangimentos causados durante a avallaçăo antropometrica. No entanto, apesar dos riscos serem

considerados minimos, para analise antropometrica e apilcaçăo dos questionarios, o pesquisador sera treinado previamente e o partidipante sera

Informado soore possivels desconfortos. Alem dsso, os pesquisadores se comprometem em fornecer 0 suporte necessano caso ocorram. Sendo

assim, caso hala necessidade, o indviduo podera ser encaminhado para o acompannamento psicoicgico no servico da dinica escola de Psicologa

da Faculdade Sarta Mara.

Benendos:

A partir da presente pesquisa possibilitara identricar os riscos psicologicos e nutincionals acs quals esses estudantes est5o vuneravels,

evidendando e fornecendo novos dados de como as thuras profissdes podem a fetar a saude desse pobilco.

\section{Comentarios $\theta$ Consideraçes sobre a Pesqulsa:}

A pesquisa esta bem delineada e coserva 05 preceitos eticos exigldos pela legislaçăo, em especial a Resoluçato 466/12.

Consideraçes sobre 08 Termos de apresentaça obrigatorla:

Todos os Termos de apresentaçăo obrigatoria foram apresentados adequadamente: Termo de Consentlmento Livre e Esclarecido (TCLE); - Folha de rosto (datada e assinada); - Termo de Compromisso e responsabilidade do pesquisador responsavel (datado e assinado); Termo de compromisso e responsabilldade do pesquisador participante (datado e assinado); - Projeto completo e Instrumento de coleta de dados.

Conclusdee ou Pendênclas e Lata de Inadequaçoes:

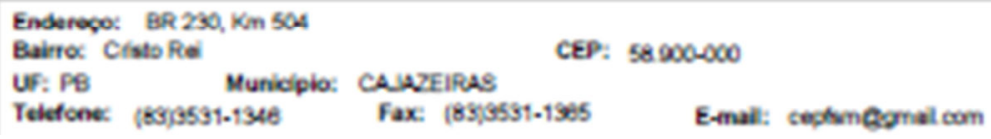




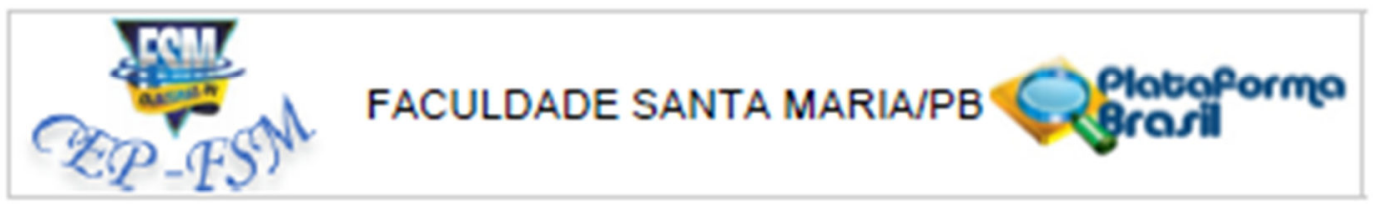

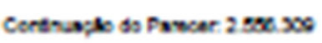

Sem pendendas elou inadequaçes.

Consideraçese Finals a criterio do CEP:

Este parecer fol elaborado bseeado nos documentos ababo relacionados:

\begin{tabular}{|c|c|c|c|c|}
\hline Tipo documento & Arquivo & Postagem & ALI: & Situaç30 \\
\hline $\begin{array}{l}\text { Informachdes Basicas } \\
\text { do Proleto }\end{array}$ & 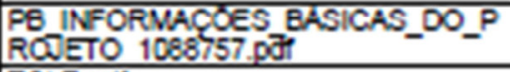 & $\begin{array}{c}06 / 03 / 2018 \\
19: 29: 10\end{array}$ & & Acelto \\
\hline $\begin{array}{l}\text { TCLE / Termos de } \\
\text { Assentimento / } \\
\text { Justilcativa de } \\
\text { Ausencla }\end{array}$ & TCLE.pơt & $\begin{array}{c}06 / 03 / 2018 \\
19: 28: 50\end{array}$ & $\begin{array}{l}\text { LARISSA DE BRTTO } \\
\text { MEDEIROS }\end{array}$ & Aceito \\
\hline Folha de Rosto & folnaDeRosto.port & $\begin{array}{c}06 / 03 / 2018 \\
19: 28: 12\end{array}$ & $\begin{array}{l}\text { LARISSA DE BFTTO } \\
\text { MEDEIROS }\end{array}$ & Acelto \\
\hline Qutros & ECAP.pd" & $\begin{array}{c}06 / 03 / 2018 \\
19: 11: 30\end{array}$ & $\begin{array}{l}\text { LARISSA DE BRTTO } \\
\text { MEDEIROS }\end{array}$ & Aceito \\
\hline Cutros & FICAA_AN.pd & $\begin{array}{c}05 / 0352018 \\
19: 10: 57\end{array}$ & $\begin{array}{l}\text { LAFSSSADEBTIO } \\
\text { MEDEIROS }\end{array}$ & Aceto \\
\hline Quiros & QUESTIONARIO_DIETAS.pCI & $\begin{array}{c}06 / 03 / 2018 \\
19: 10: 37\end{array}$ & $\begin{array}{l}\text { LAFISSA DE BRTTO } \\
\text { MEDEIROS }\end{array}$ & Aceito \\
\hline Qutros & anuenciafSM pdr & $\begin{array}{c}06 / 03 / 2018 \\
19: 10: 00\end{array}$ & $\begin{array}{l}\text { LARISSA DE BFTOO } \\
\text { MEDEIROS }\end{array}$ & Aceito \\
\hline Qufros & anuendaFAFIC. pdt & $\begin{array}{c}06 / 03 / 2018 \\
19: 09: 42\end{array}$ & $\begin{array}{l}\text { LARISSA DE BRTTO } \\
\text { MEDEIROS }\end{array}$ & Acelto \\
\hline $\begin{array}{l}\text { Projeto Detainaco / } \\
\text { Brochura } \\
\text { Investlgador }\end{array}$ & PROJETO_DANIELLE. $O O C X$ & $\begin{array}{c}06 / 03 / 2018 \\
19: 09: 23\end{array}$ & $\begin{array}{l}\text { LARISSA DE BFTTO } \\
\text { MEDEIROS }\end{array}$ & Aceito \\
\hline Crçamento & ORCAMENTO.PO' & $\begin{array}{c}06 / 03 / 2018 \\
19: 07: 56\end{array}$ & $\begin{array}{l}\text { LARISSA DE BRTO } \\
\text { MEDEIRQS }\end{array}$ & Acelto \\
\hline Cronograma & CFIONOGRAMA PCR & $\begin{array}{c}06 / 03 / 2018 \\
19: 07: 46\end{array}$ & $\begin{array}{l}\text { LAFISSA DE BRTTO } \\
\text { MEDEIROS }\end{array}$ & Aceito \\
\hline $\begin{array}{l}\text { Declaraç3o de } \\
\text { Pesquisadores }\end{array}$ & TCR_responsave.pdr & $\begin{array}{c}06 / 03 / 2018 \\
19: 07: 35\end{array}$ & $\begin{array}{l}\text { LAFISSA DE BFTTO } \\
\text { MEDEIROS }\end{array}$ & Aceito \\
\hline $\begin{array}{l}\text { Decaraçao de } \\
\text { Pesquisadores }\end{array}$ & TCR_partiparte.por & $\begin{array}{c}05003 / 2018 \\
19: 07: 22\end{array}$ & $\begin{array}{l}\text { DAFUSSADEBTIO } \\
\text { MEDEIROS }\end{array}$ & Aceto \\
\hline
\end{tabular}

SItuaç3o do Parecer:

Aprovado

Necessita Apreclaça da CONEP:

NEO

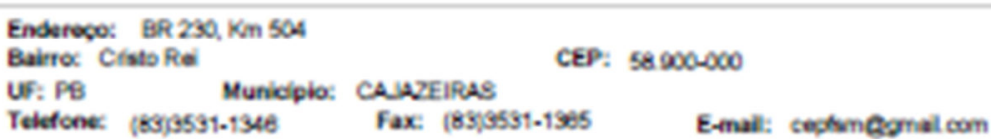

